\title{
Neoplasia Cervical Intraepitelial
}

\author{
HOSPITAL UNIVERSITARIO DE CALDAS \\ Septiembre 1985 - Febrero 1987
}

Dres: Carlos Alberto Esparza D.*, Mario Calle Mesa**

\section{RESUMEN}

Se revisaron 143 historias clínicas, correspondientes a pacientes que asistieron por primera vez a la consulta de detección precoz de cáncer del cervix en el Hospital Universitario de Caldas, en el período comprendido entre el 1 de septiembre de 1985 y el 28 de febrero de 1987 y tuvieron diagnóstico de NIC.

El objetivo principal, fue evaluar lo que se ha realizado en dicho programa, cuantificando y caracterizando la experiencia mediante estadísticas descriptivas. Se encontró una edad promedio de 37,2 años. El $73,4 \%$ procedían de área urbana. Su paridad promedio fue de 5.7 embarazos. 131 pacientes fueron remitidas a esta consulta por citología Grado III o mayor. A 100 de ellas se les practicó solamente una colposcopia. A las 43 restantes dos o más. Se correlacionaron los hallazgos de la primera colposcopia con las citologías de remisión y con el diagnóstico definitivo, así como también las lesiones encontradas en la primera colposcopia con el diagnóstico final. Se analizaron los tratamientos de acuerdo con dicho diagnóstico.

\section{INTRODUCCION}

En nuestro estudio sobre Diagnóstico Precoz de Cáncer de Cervix (1), anunciamos la presente revisión sobre el subgrupo de pacientes en quienes

\footnotetext{
* Profesor Asociado U. de Caldas

** Profesor Titular U. de Caldas
}

se hizo un diagnóstico definitivo de neoplasia intraepitelial cervical (NIC).

En dicho estudio encontramos un incremento muy notable en el diagnóstico de NIC a partir de 1985, en la consulta externa de Ginecología de nuestro Hospital.

Se acepta, en general, que el diagnóstico y la erradicación de la NIC previene el desarrollo de cáncer invasor de cervix, $(2,4)$ y por consiguiente, disminuye la mortalidad por esta enfermedad. Esto no ha ocurrido en nuestro país y desde 1975 se han hecho críticas a la utilización del recurso de la citología. (4)

Consideramos que tiene especial importancia analizar los resultados de las actividades desarrolladas con este grupo específico de pacientes en el programa de detección precoz de cáncer de cervix del Hospital Universitario de Caldas en el período de 18 meses, comprendidos entre el 1 de septiembre de 1985 y el 28 de febrero de 1987. Tanto la teoría como la práctica clínica en este campo se han desarrollado notablemente, incrementando su complejidad, tecnología, costos, molestias y riesgos potenciales para los pacientes.

Compararemos nuestros resultados con los que encontraron Vélez y Mejía, en su trabajo para optar al título de Especialistas, intitulado Neoplasia Cervical Intraepitelial y Carcinoma del Cervix, Hospital Universitario de Caldas, 1980 - 1984, presentado en 1986 (5). Los analizaremos también a la luz de otros datos publicados en la literatura. 


\section{MATERIALES Y METODOS}

Uno de los autores (Doctor Mario Calle Mesa), revisó cuidadosamente cada una de las historias clínicas correspondientes a las pacientes que asistieron al programa en el período mencionado.

La información contenida en las historias clínicas, fue registrada por el investigador en formularios precodificados y diseñados por él mismo para NIC y CANCER DE CERVIX. Cada formulario contiene 56 items o variables y aproximadamente 178 códigos.

Estos datos se archivaron en disquetes de 5,25 pulgadas mediante un microcomputador NEC PC-8801A. Posteriormente, se procesaron utilizando programas especiales y se obtuvo la información necesaria para el logro de los objetivos específicos

\section{RESULTADOS Y DISCUSION}

\subsection{RESULTADOS}

TABLA 1

EDAD DE LOS PACIENTES

\begin{tabular}{|lcr|}
\hline Edad & Número de Casos & $\%$ \\
\hline $11-15$ & 1 & 0,7 \\
$16-20$ & 2 & 1,4 \\
$21-25$ & 12 & 8,4 \\
$26-30$ & 27 & 18,9 \\
$31-35$ & 25 & 17,5 \\
$36-40$ & 29 & 20,3 \\
$41-45$ & 19 & 13,3 \\
$46-50$ & 11 & 7,7 \\
$51-55$ & 10 & 7,0 \\
$56-60$ & 3 & 2,1 \\
$61-65$ & 2 & 1,4 \\
$66-70$ & 1 & 0,7 \\
$71-75$ & 1 & 0,7 \\
\hline Total & 143 & 100,0 \\
\hline
\end{tabular}

Observamos que el 70,0\% de las pacientes se encuentra en el grupo de edad comprendido entre 26 y 45 años. La edad promedio fue de 37,2 años con una D.S. de 10,5. del estudio. Tanto para el archivo de los datos como para la programación del procesamiento de los mismos se empleó el dBASE II, versión 2.4.

De las 300 historias clínicas revisadas en nuestro estudio anterior se seleccionaron 143 correspondientes a pacientes con diagnóstico definitivo de neoplasia intraepitelial cervical (NIC).

Para el análisis de la información se emplearon estadísticas sencillas que incluyen porcentajes, promedios para datos agrupados y no agrupados y desviación estandar.

Se trata de un estudio retrospectivo, descriptivo, que intenta caracterizar cuantitativamente al grupo de pacientes atendidas en esta consulta subespecializada.

\section{FIGURA 1}

EDAD DE LAS PACIENTES

\begin{tabular}{|l|l|l|l|l|l|l|l|l|l|}
\hline \hline 3 & 39 & 54 & 30 & 13 & 3 & 1 \\
\hline
\end{tabular}

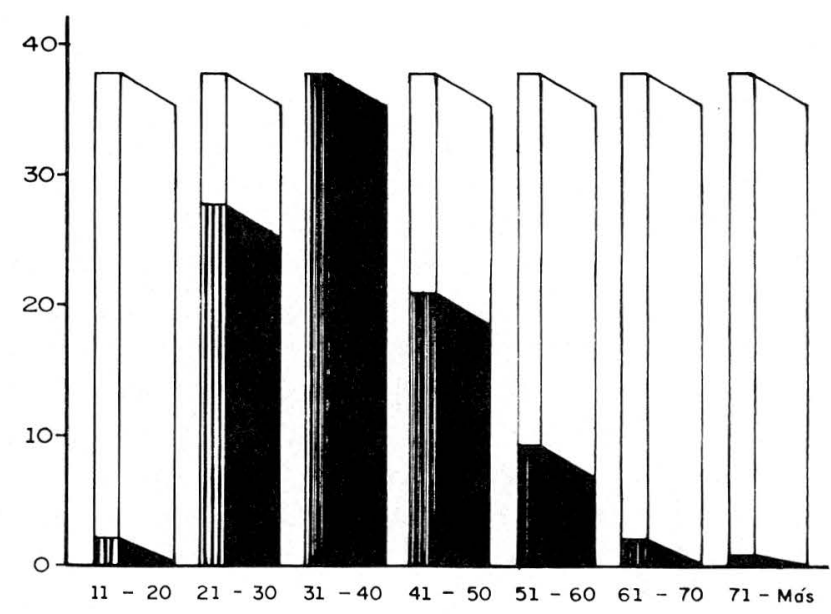

TABLA 2

ESTADO CIVIL

\begin{tabular}{|lcr|}
\hline Estado Civil & Número de casos & $\%$ \\
\hline Solteras & 12 & 8,4 \\
Casadas & 96 & 67,1 \\
Unión libre & 25 & 17,5 \\
Viudas o separadas & 10 & 7,0 \\
\hline Total & 143 & 100,0 \\
\hline
\end{tabular}


FIGURA 2

ESTADO CIVIL

\begin{tabular}{|l|l|l|l|l|l|}
\hline \multicolumn{5}{|c|}{ NUMERO DE CASOS } \\
\hline 12 & & 96 & 25 & 10 \\
\hline
\end{tabular}

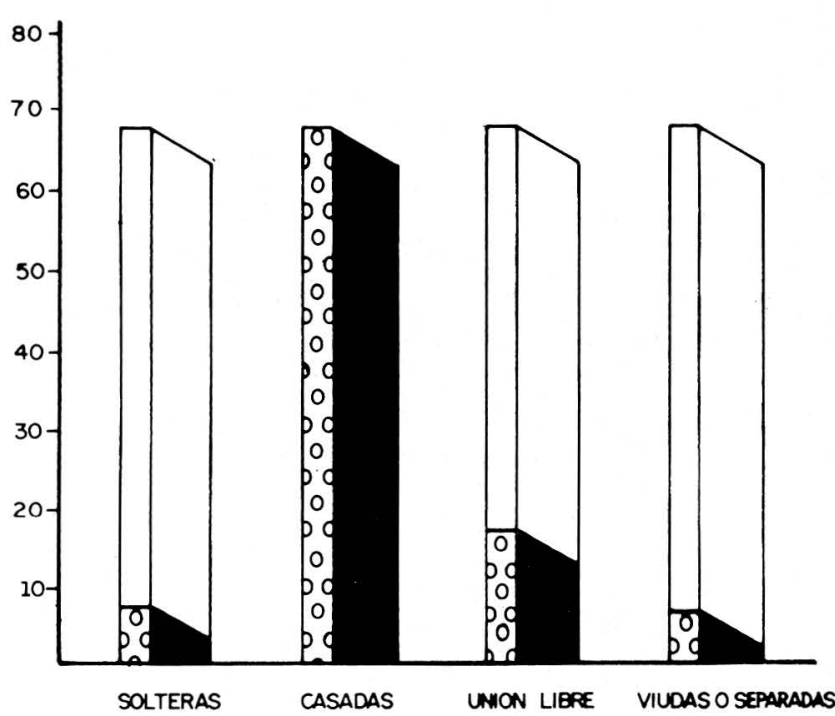

TABLA 3

NIVEL DE INSTR UCCION

\begin{tabular}{|lrr|}
\hline Nivel & Número de casos & $\%$ \\
\hline Primaria completa & 16 & 11,2 \\
Primaria incompleta & 47 & 32,9 \\
Secundaria completa & 3 & 2,1 \\
Secundaria incompleta & 16 & 11,2 \\
Postsecundaria & 2 & 1,4 \\
Sin dato & 55 & 38,5 \\
Analfabetas & 4 & 2,8 \\
\hline Total & 143 & 100,0 \\
\hline
\end{tabular}

TABLA 4

DOMICILIO

\begin{tabular}{|lrr|}
\hline Domicilio & Número de casos & $\%$ \\
\hline Urbano & 105 & 73,4 \\
Rural & 38 & 26,6 \\
\hline Total & 143 & 100,0 \\
\hline
\end{tabular}

E1 73,4\% de los casos, corresponde a pacientes procedentes de área urbana.
FIGURA 3

NIVEL DE INSTRUCCION

\begin{tabular}{|l|l|l|l|l|l|l|l|l|}
\hline \multicolumn{7}{|c|}{ NUMERO } & DE & CASOS \\
\hline 16 & & 47 & 3 & 16 & 2 & 55 & 4 \\
\hline
\end{tabular}

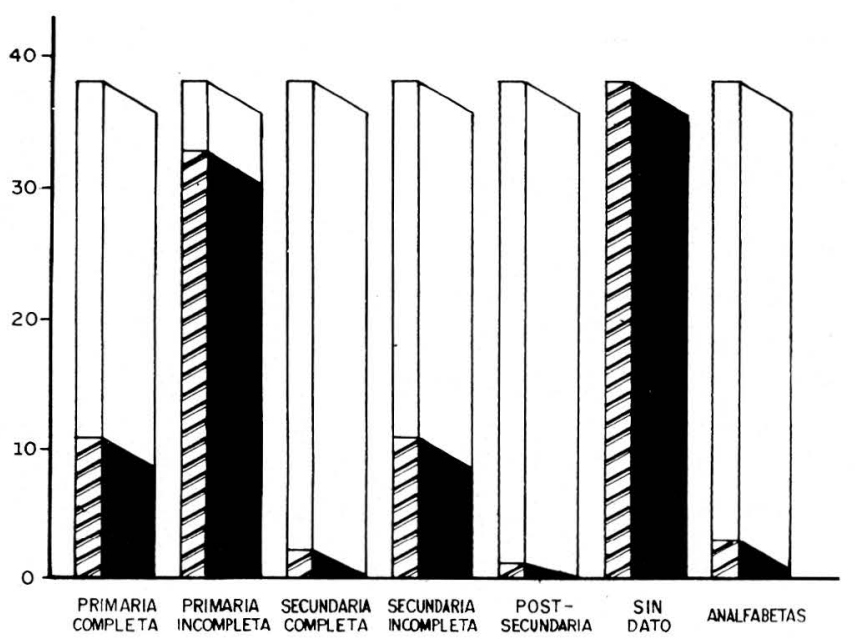

FIGURA 4

DOMICILIO

\begin{tabular}{|l|l|l|}
\hline \multicolumn{2}{|c|}{ NUMERO DE CASOS } \\
\hline 38 & & 105 \\
\hline
\end{tabular}

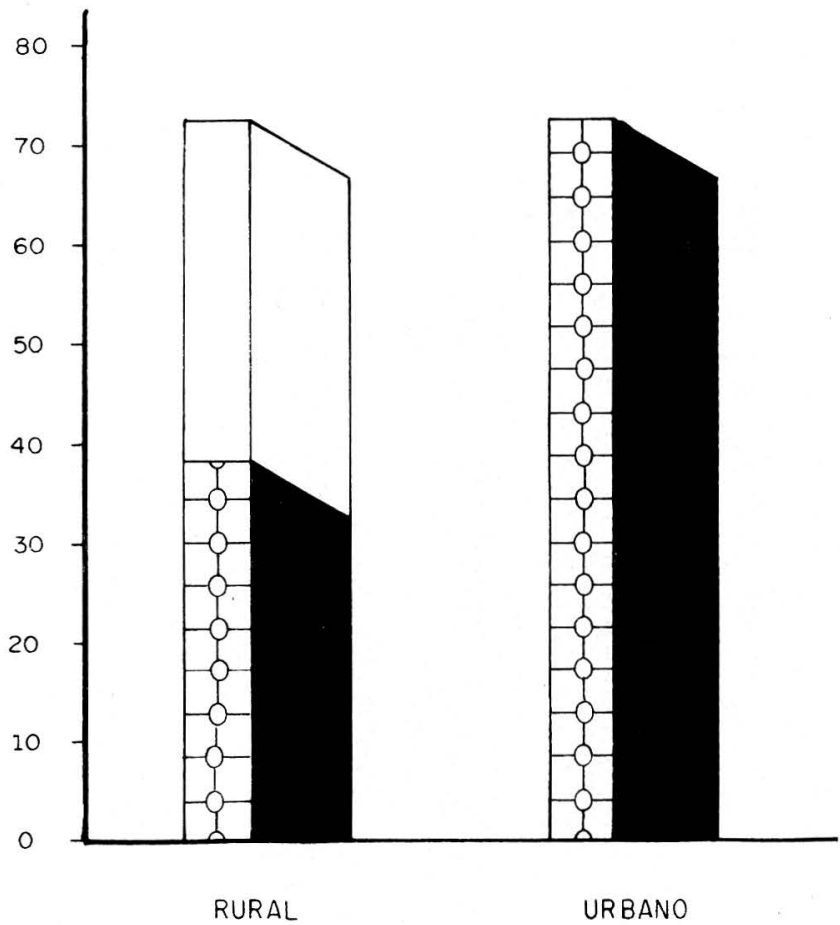


TABLA 5

\section{DISTRIBUCION SEGUN PARIDAD}

\begin{tabular}{|lcr|}
\hline Número embarazos & Número de casos & $\%$ \\
\hline Uno & 9 & 6,3 \\
Dos & 19 & 13,3 \\
Tres & 24 & 16,8 \\
Cuatro & 21 & 14,7 \\
Cinco & 16 & 11,2 \\
Seis & 6 & 4,2 \\
Siete & 6 & 4,2 \\
Ocho o más & 41 & 28,7 \\
Sin dato & 1 & 0,7 \\
\hline Total & 143 & 100,0 \\
\hline
\end{tabular}

Promedio 5,7 embarazos, D.S. 3,9

FIGURA 5

DISTRIBUCION SEGUN PARIDAD

\begin{tabular}{|l|l|l|l|l|}
\hline \multicolumn{5}{|c|}{ NUMERO DE CASOS } \\
\hline 52 & 43 & 47 & 1 \\
\hline
\end{tabular}

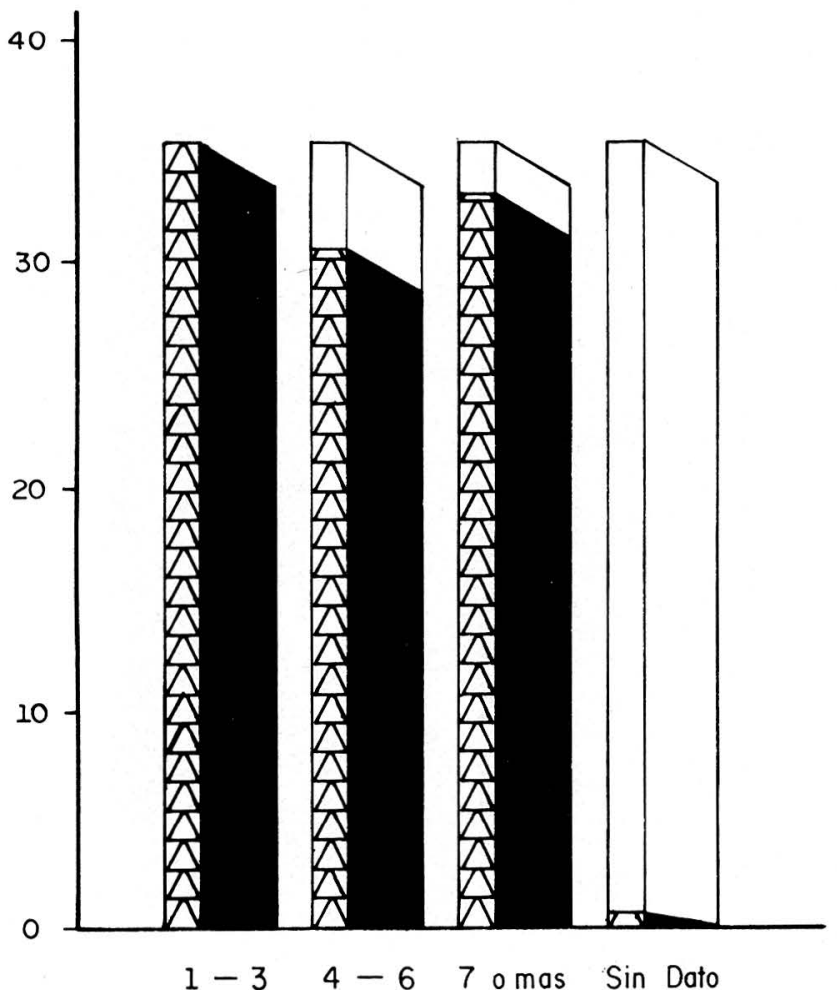

TABLA 6

CONSUMO DE CIGARRILLOS

\begin{tabular}{|lrr|}
\hline $\begin{array}{c}\text { Número de cigarrillos } \\
\text { por día }\end{array}$ & Número de casos & $\%$ \\
\hline Ninguno & 51 & 35,6 \\
$1-10$ & 16 & 11.2 \\
$11-20$ & 5 & 3,5 \\
21 ó más & 1 & 0,7 \\
Sin dato & 70 & 49,0 \\
\hline Total & 143 & 100,0 \\
\hline
\end{tabular}

Llama la atención el gran porcentaje de pacientes, $49,0 \%$ que carecen de esta información en sus historias clínicas.

FIGURA 6

CONSUMO DE CIGARRILLOS

\begin{tabular}{|l|c|c|c|c|c|}
\hline \multicolumn{5}{|c|}{ NUMERO } & \multicolumn{4}{c|}{ DE CASOS } \\
\hline 51 & 16 & 16 & 5 & 1 & 70 \\
\hline
\end{tabular}

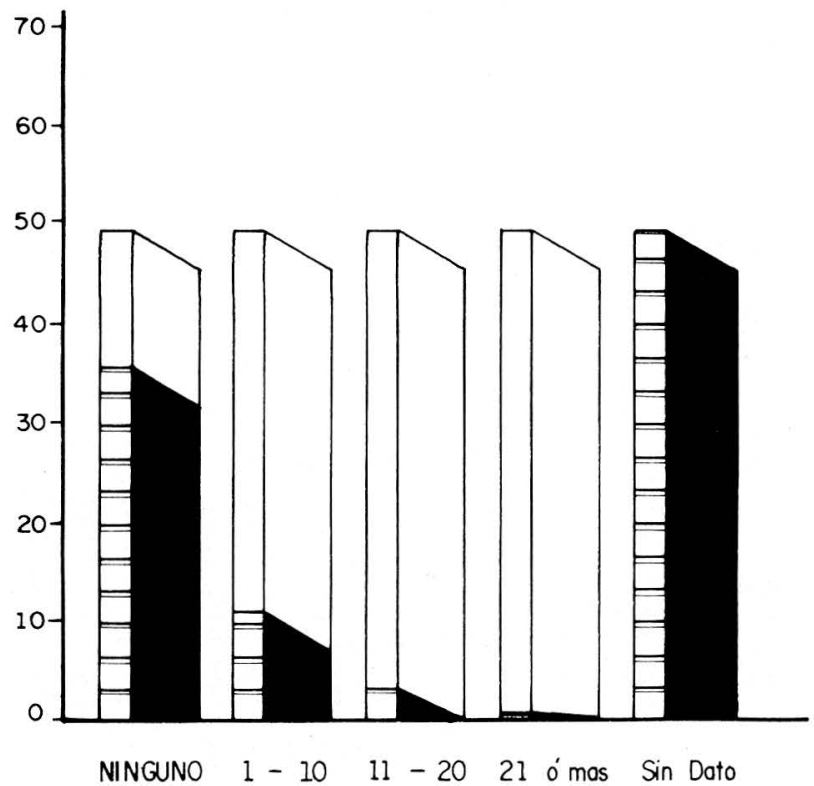


TABLA 7

DISTRIBUCION SEGUN PAP DE REMISION

\begin{tabular}{|lcr|}
\hline PAP & Número de casos & $\%$ \\
\hline II & 12 & 8,4 \\
III & 89 & 62,2 \\
IV & 29 & 20,3 \\
V & 3 & 2,1 \\
Sin PAP & 10 & 7,0 \\
\hline Total & 143 & 100,0 \\
\hline
\end{tabular}

El $84,6 \%$ de las pacientes fueron remitidas a la consulta por presentar PAP III o mayor. Observamos que hubo 10 casos que no acudieron a la consulta por citologías anormales, sino por otras razones, tales como diagnósticos de NIC hechos mediante biopsias tomadas al azar, hemorragias postmenopáusicas, etc.

FIGURA 7

\section{DISTRIBUCION SEGUN PAP DE REMISION}

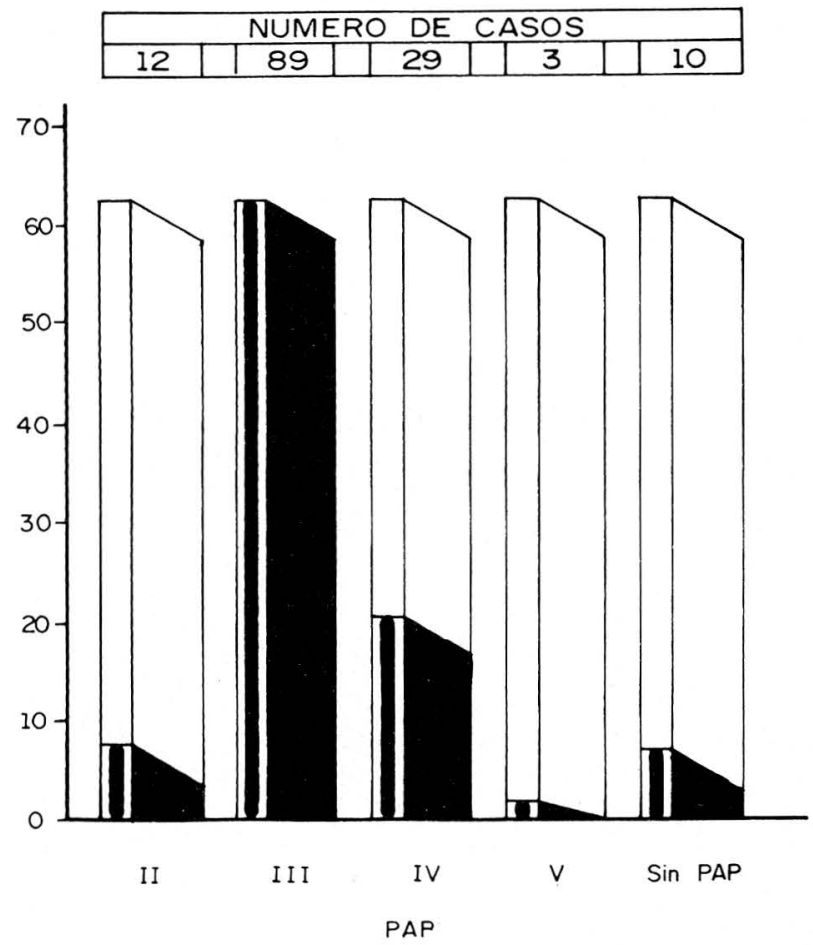

TABLA 8

DISTRIBUCION SEGUN NUMERO DE COLPOSCOPIAS

\begin{tabular}{|ccc|}
\hline $\begin{array}{c}\text { Número } \\
\text { colposcopias }\end{array}$ & $\begin{array}{c}\text { Número } \\
\text { de casos }\end{array}$ & $\%$ \\
\hline 1 & 100 & 69,9 \\
2 & 31 & 21,7 \\
3 & 10 & 7,0 \\
4 & 0 & 0,0 \\
5 & 2 & 1,4 \\
\hline Total & 143 & 100,0 \\
\hline
\end{tabular}

En 30,1\% de los casos se repitió la colposcopia una o más veces. El número total de colposcopias practicadas a las 143 pacientes fue de 202 .

FIGURA 8

DISTRIBUCION SEGUN NUMERO DE COLPOSCOPIAS

\begin{tabular}{|l|l|l|l|l|l|l|}
\hline \multicolumn{6}{|c|}{ NUMERO DE CASOS } \\
\hline 100 & 31 & 10 & 0 & 2 \\
\hline
\end{tabular}

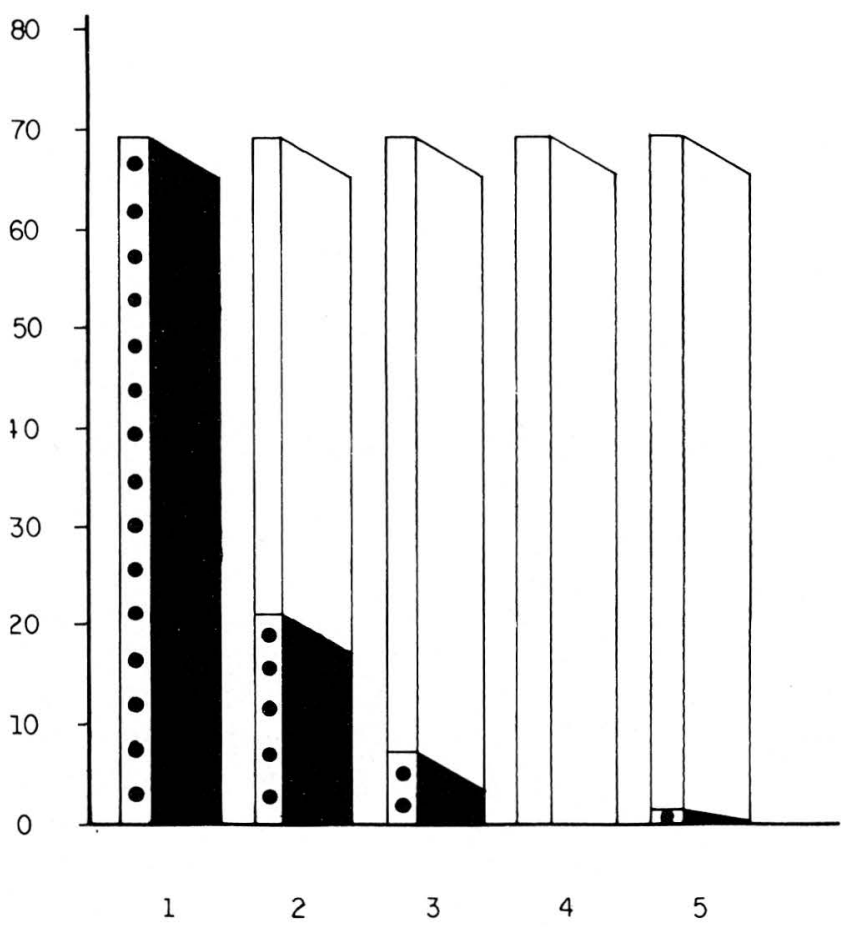


TABLA 9

HALLAZGOS PRIMERA COLPOSCOPIA SEGUN PAP DE REMISION

\begin{tabular}{|lcccccc|}
\hline PAP & Normal & Area acetoblanca & Mosaico & Punteado & Leucoplasia & Vasos Atípicos \\
\hline II & 0 & 3 & 3 & 5 & 4 & 2 \\
III & 15 & 31 & 16 & 26 & 32 & 8 \\
IV & 5 & 20 & 6 & 10 & 6 & 0 \\
V & 0 & 2 & 0 & 0 & 2 & 22 \\
\hline Total * - & $20 * *$ & 56 & 25 & 41 & 44 & 8 \\
\hline
\end{tabular}

* Corresponde al número de veces que ocurre una lesión. En una misma colposcopia pueden presentarse dos o más lesiones.

** No se incluye una colposcopia normal en una paciente sin PAP de remisión, cualquier lesión colposcópica puede encontrarse en casos de citologías grado II o mayor. Sin embargo, con PAP III, son más frecuentes, por su orden, leucoplasia, área acetoblanca y punteado. Con PAP IV, área acetoblanca y punteado.

TABLA 10

HALLAZGOS PRIMERA COLPOSCOPIA EN RELACION CON DIAGNOSTICO HISTOPATOLOGICO DEFINITIVO

FIGURA 9

\section{HALLAZGOS PRIMERA COLPOSCOPIA SEGUN PAP REMISION}

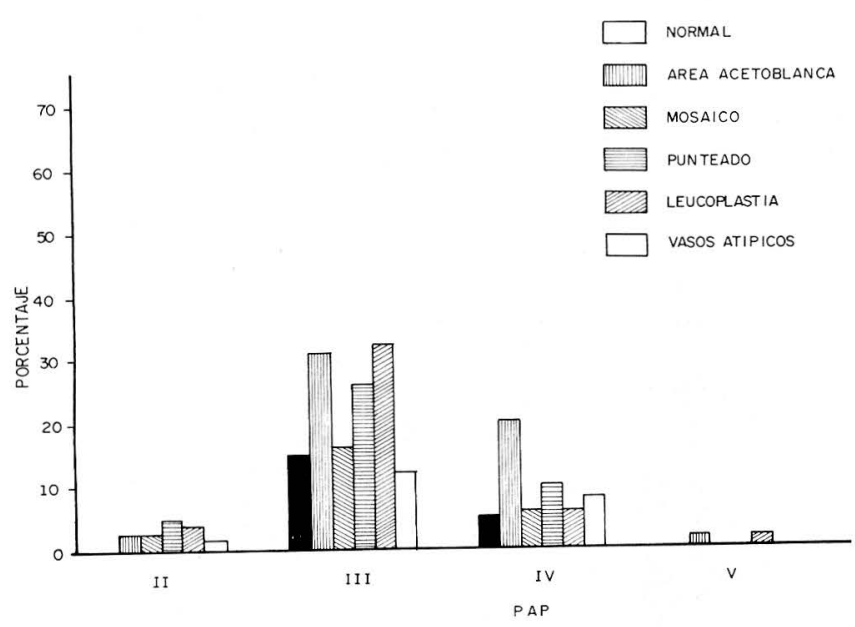

Cada uno, de dos colposcopistas, realizó 10 de las 20 colposcopias normales. 18 de ellas fueron satisfactorias. A 18 se les realizó una segunda colposcopia: Cuatro resultaron de nuevo normales. 13 presentaron alguna lesión. Una paciente sin datos en la Historia Clínica.

Nueve pacientes fueron sometidas a tercera colposcopia: tres continuaron siendo normales; seis presentaron una o dos lesiones.

\begin{tabular}{|lrrrr|}
\hline $\begin{array}{l}\text { Diagnóstico } \\
\text { Lesión }\end{array}$ & NIC I & NIC II & NIC III & Total * \\
\hline Acetoblanca & 10 & 13 & 38 & 61 \\
Mosaico & 4 & 5 & 17 & 26 \\
Punteado & 4 & 9 & 31 & 44 \\
Leucoplasia & 5 & 8 & 34 & 47 \\
Vasos atípicos & 3 & 4 & 17 & 24 \\
Normal & 2 & 5 & 14 & 21 \\
\hline
\end{tabular}

* Número de veces que se presentó la lesión en la primera colposcopia. En una misma colposcopia pueden ocurrir dos o más lesiones.

\section{FIGURA 10}

HALLAZGOS PRIMERA COLPOSCOPIA EN RELACION CON DIAGNOSTICO HISTOPATOLOGICO DEFINITIVO

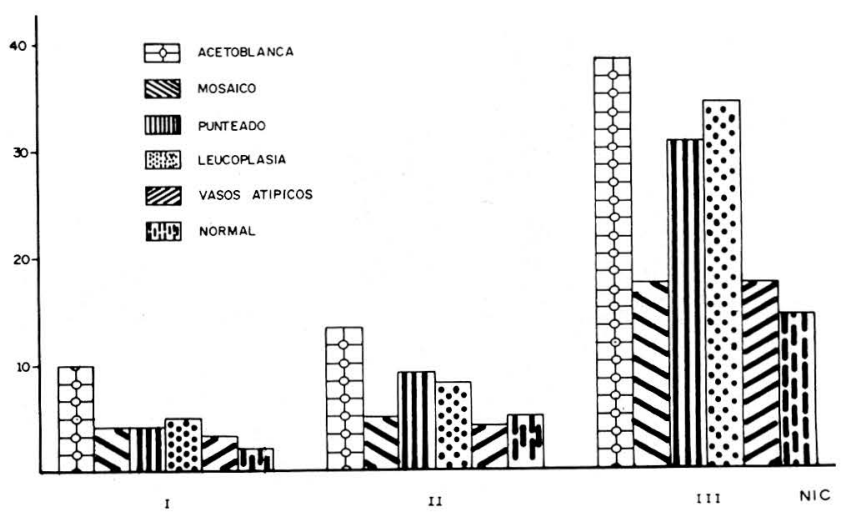


TABLA 11

DIAGNOSTICO DEFINITIVO SEGUN PAP DE REMISION

\begin{tabular}{|lllllll|}
\hline $\begin{array}{c}\text { PAP } \\
\text { Diagnóstico }\end{array}$ & II & III & IV & V & Total & $\%$ \\
\hline NIC I & 2 & 11 & 3 & 0 & 16 & 12,0 \\
NIC II & 3 & 21 & 3 & 0 & 27 & 20,3 \\
NIC III & 7 & 57 & 23 & 3 & 90 & 67,7 \\
\hline Total * & 12 & 89 & 29 & 3 & 133 & 100,0 \\
\hline
\end{tabular}

* Número de pacientes en cada grupo de PAP

Edad promedio de las pacientes con NIC I : 38,9 Edad promedio de las pacientes con NIC II: 33,9 Edad promedio de las pacientes con NIC III: 38,0

En estas cifras están incluidas las 10 pacientes que no tuvieron PAP de remisión.

FIGURA 11

\section{DIAGNOSTICO DEFINITIVO SEGUN PAP DE REMISION}

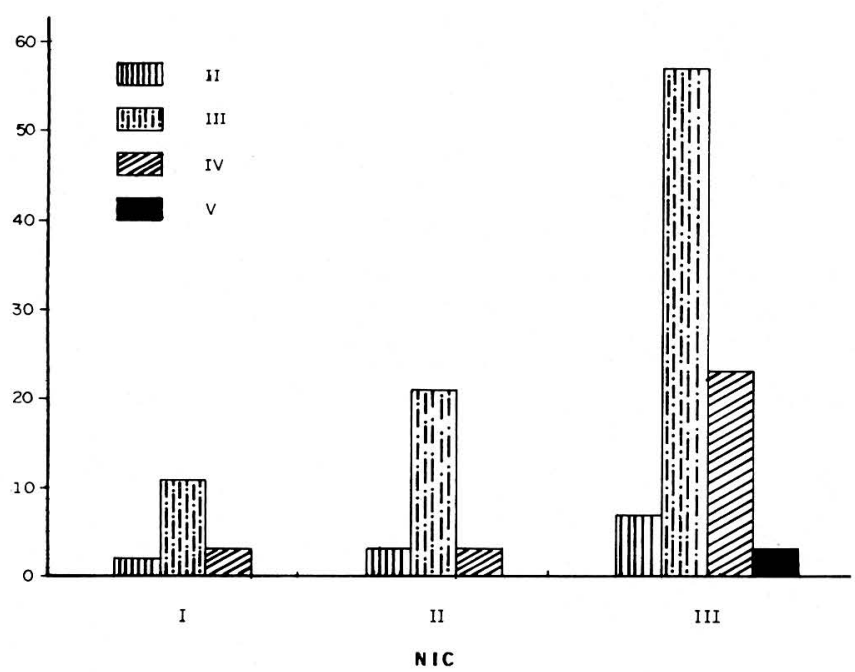

\subsection{DISCUSION}

Vélez y Mejía (5). encontraron en el período 1980 - 1984, un promedio anual de consultas ginecológicas nuevas de 1134, de las cuales 43 correspondieron a casos de NIC. Es decir, que éstos representaron el 3,8\% de las consultas nuevas, en promedio.
TABLA 12

TRATAMIENTO SEGUN DIAGNOSTICO

\begin{tabular}{|lcccccr|}
\hline $\begin{array}{l}\text { Diagnóstico } \\
\text { Tratamiento }\end{array}$ & NIC & I & NIC II & NIC III & Total* & $\%$ \\
\hline $\begin{array}{l}\text { Observación } \\
\text { Criocirugía }\end{array}$ & 12 & 7 & 5 & 24 & 16,8 \\
Radioterapia & & 16 & 0 & 22 & 15,4 \\
$\begin{array}{l}\text { Conización } \\
\text { Histerectomia }\end{array}$ & 0 & 4 & 38 & 42 & 29,4 \\
$\begin{array}{l}\text { Vaginal } \\
\text { Histerectomia }\end{array}$ & $1 * *$ & & 1 & 0,7 \\
$\begin{array}{l}\text { ampliada } \\
\text { Anexohisterectomia }\end{array}$ & 1 & 21 & 22 & 15,4 \\
Histerolinfadenectomía & & 28 & 30 & 21,0 \\
\hline Total ***** & 18 & 31 & $94 * * *$ & 1 & 0,7 \\
\hline
\end{tabular}

* Total tratamientos

** Alto riesgo quirúrgico

*** Prolapso genital

**** 26 años. Tratamiento aparentemente equivocado

***** Total de casos de NIC

FIGURA 12

TRATAMIENTO SEGUN DIAGNOSTICO

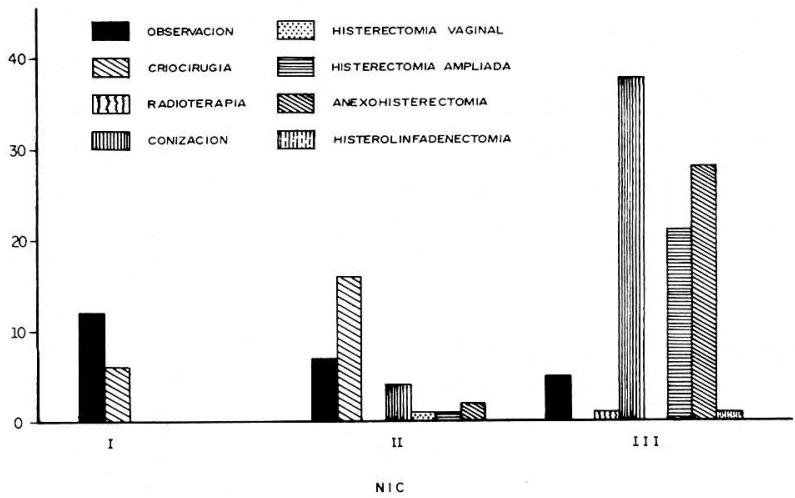

En nuestro estudio encontramos 1811 consultas ginecológicas nuevas por año, de las cuales 95 correspondieron a pacientes con NIC. Estas representaron el $5,2 \%$ en promedio.

Mientras que el incremento en el total de consultas nuevas fue de $59,7 \%$, el de casos nuevos de NIC fue el $120,9 \%$. Es claro que ocurrió un au- 
mento muy notable en la incidencia de esta patología en nuestro hospital.

En el estudio anterior presentamos la explicación más sencilla de este hecho: mayor interés y mayores recursos dedicados al diagnóstico precoz de cáncer preclínico de cervix, desde septiembre de 1985.

Vélez y Mejía (5), encontraron una edad promedio de 41,5 años en las pacientes con NIC. En nuestro estudio dicha edad fue de 37,2 años, con una mínima de 15 y una máxima de 73 .

En el período 1980 - 1984, se encontró como edad promedio para las pacientes con NIC 41,5 años. Para 212 casos de cáncer invasor, en cambio, el promedio de edad fue de 48,1 vale decir, 6,6 años más. El 86,8\% de las pacientes con cáncer invasor fueron mayores de 35 años. Solamente el 13,2 fueron menores de 34 .

En el presente estudio, la edad promedio de pacientes con NIC fue de 37,2 años. Esto es, 10,9 años menos que el promedio de las pacientes con cáncer invasor en el período 1980 - 1984.

Si consideramos por separado la edad promedio de las pacientes con NIC I (38,9 años), NIC II (33,9 años), NIC III (38,0 años), llama la atención el hecho de que aparentemente es más joven el grupo de NIC II que el de NIC I. Esto no concuerda con la teoría de que se trata de un continuo que empieza en displasia leve y continua con ca in situ, para luego terminar en el cáncer invasor. Pudiera quizás encontrarse una explicación de este fenómeno en el escaso número de pacientes en cada grupo y en que coincidencialmente las edades extremas en el grupo de NIC I (37 y 61) son mayores que en el de NIC II ( 19 y 58 ).

Las observaciones previas sobre edad coinciden bastante con las de Stiernsword y Cols. (4) en el sentido de que más del $90 \%$ de los cánceres invasores ocurren en mujeres mayores de 35 años y que el recurso citológico se emplea preferentemente en mujeres más jóvenes que acuden a los programas de planificación familiar y provenientes de áreas urbanas. Más aún, se lo utiliza en "volver a explorar a mujeres jóvenes a las que se ha diagnosticado displasia del cuello uterino". En nuestro estudio pudimos revisar 146 citologías de control, encontrando que el intervalo promedio entre ellas es de 4,4 meses.
Parece surgir aquí una controversia importante entre subespecialistas oncólogos, ginecólogos y epidemiólogos. Aquellos tratando de aplicar las más refinadas prácticas de la medicina norteamericana y canadiense a nuestro medio y éstos tratando de impulsar la filosofía de Alma Ata, de salud para todos en el año 2000. Si de lo que se trata es de disminuir las tasas de mortalidad por cáncer de cervix, es muy improbable que lo logremos, si continúan las prácticas actuales en el uso de la citología. Es más; qué sentido tiene continuar aplicando cada trimestre una prueba de tamizaje a pacientes en quienes ya se ha establecido el diagnóstico de NIC? Estamos plenamente de acuerdo con Stiernsward y Cols, cuando afirman que "no es realista imitar los programas de detección de los países desarrollados". En la India, “aún esfórzandose por ampliar los servicios de citología, para el año 2000 sólo se podrán hacer exámenes de detección al 25\% de las mujeres más expuestas". Con nuevos criterios, menos subespecializados, pero más epidemiológicos, se obtendría " $60 \%$ de la eficacia alcanzable con recursos limitados. .. con una probabilidad razonable de reducir la mortalidad".

Es necesario reconsiderar las acciones de diagnóstico precoz de cáncer de cervix, de suerte que ellas lleguen a mayor número de pacientes con factores de riesgo, así como también a las de áreas rurales. La Organización Panamericana de la Salud (OPS) encontró en 1984, en programas de varios países de América Latina, "que la población objeto de la citología es limitada, hay exceso de citologías no necesarias en el mismo grupo de mujeres, generalmente usuarias de planificación familiar y de servicios privados. . ." Restrepo et al, señalan que "la recomendación de realizar citologías cada seis meses es otro ejemplo de la distorsión de las normas. Finalmente, se debe señalar que el exceso de comercialización de la técnica de citología está ocasionando su mal uso y desvirtuando la práctica de los técnicos que la realizan" (3).

La mayor parte de las citologías de remisión fueron grado III $(62,2 \%)$ y 57 de ellas $(64 \%)$ terminaron con diagnóstico definitivo de NIC III. Aparentemente no hay una buena correlación, puesto que sería de esperar que la mayor parte de ellas hubieran correspondido a NIC I y II. ¿ंHay alguna falla en el diagnóstico de citotécnicos y de citopatólogos? No tenemos elementos para contestar a esta pregunta, pero vale la pena destacar el hecho para analizarlo más a fondo. 
La correlación es mucho mejor para el PAP IV que en el 79,3\% de los casos correspondió a NIC III.

En el caso de PAP V la correlación fue excelente, pero los casos muy pocos, para poder sacar conclusión alguna válida.

Desde el punto de vista de correlación entre citología y hallazgos o lesiones colposcópicas, encontramos 20 pacientes con citologías III y IV cuya primera colposcopia fue normal y que terminaron con un diagnóstico definitivo de NIC. Corresponden a un $14,0 \%$ del total de 143 pacientes.

En cuanto al tratamiento observamos que en el $44.8 \%$ de las pacientes se emplearon tratamientos conservadores (criocirugía 22 casos y conización 42) y cirugías mayores o radioterapia en el $39,2 \%$.

La criocirugía se utilizó principalmente en pacientes con NIC II y la conización en casos de NIC III.

Una paciente fue tratada sólo con radioterapia porque se consideró de alto riesgo para cirugía.

Cuatro de las cinco pacientes con NIC III sometidas a observación, recibieron tal manejo debido a que se encontraban en embarazo.

Llama la atención al subgrupo de 28 pacientes tratadas con anexohisterectomía. Tres eran menores de 40 años. Las restantes mayores. Cabe preguntarse si hay o no sobretratamiento. La respuesta requeriría revisión de estos 28 casos, pero este no es el objetivo de nuestro estudio.
La paciente con NIC III tratada con histerolinfadenectomía, aparentemente constituye un caso de error lamentable, cuya explicación no aparece con absoluta claridad en la historia clínica.

\section{CONCLUSIONES}

1. Las pacientes con NIC, constituyeron el 5,2\% de las consultas ginecológicas nuevas, en el período de estudio. Con relación al período 1980 - 1984, hubo un incremento en el diagnóstico de $120,9 \%$.

2. La edad promedio de las pacientes fue de 37,2 años. Esto es, 10,9 años menos que el promedio de las pacientes con cáncer invasor, estudiadas en el período 1980 - 1984.

3. Planteamos la duda sobre si el recurso citológico se está empleando racionalmente en nuestro medio, si aceptamos que la meta en la campaña de diagnóstico precoz de cáncer de cervix es lograr una reducción real de la mortalidad, por esta enfermedad.

4. No encontramos buena correlación entre citologías grado III y diagnóstico definitivo de NIC III. Convendría analizar mejor las implicaciones de este hallazgo.

5. Encontramos que en el $14.0 \%$ de este grupo de pacientes, con citologías de remisión grado III y IV, la primera colposcopia fue normal, es decir, mostró una mala correlación con las citologías y con el diagnóstico final de NIC.

6. Las pacientes embarazadas con NIC, constituyeron el 2,8\% del grupo estudiado.

\section{BIBLIOGRAFIA}

1. CALLE M. y ESPARZA. Diagnóstico precoz de cáncer de cervix. Hospital Universitario de Caldas. Septiembre 1985 - Febrero 1987 s. p.

2. NELSON JH. et al. Dysplasia. Carcinoma in Situ, and Early Invasive Cervical Carcinoma CA-A Cáncer Journal for clinicians 34: 306 November/December, 1984.

3. RESTREPO HE.y COLS. Epidemiología y control del cáncer del cuello uterino en América Latina y el Caribe. Bol. Oficina Panamericana 102: 578, 1987.
4. STIERNSWARD S. y COLS. Reorientación de los exámenes de detección del cáncer cervicouterino en los países en desarrollo. Foro Mundial de la Salud 8:41, 1987.

5. VELEZ JE. y MEJIA LA. Neoplasia cervical intraepitelial del cervix. Hospital Universitario de Caldas 1980 - 1984. Tesis de Grado, 1986. 